\title{
GLUTEUS MAXIMUS AND SOLEUS COMPENSATE FOR SIMULATED QUADRICEPS ATROPHY AND ACTIVATION FAILURE DURING WALKING
}

\author{
Julie A. Thompson ${ }^{1}$, Ajit M.W. Chaudhari ${ }^{1,2,3,4}$, Laura C. Schmitt ${ }^{3,4}$, Thomas M. Best ${ }^{3,5}$ \\ and Robert A. Siston ${ }^{1,2,4}$
}

\footnotetext{
${ }^{1}$ Department of Mechanical and Aerospace Engineering, The Ohio State University, Columbus, $\mathrm{OH}$, USA

${ }^{2}$ Department of Orthopaedics, The Ohio State University, Columbus, OH, USA

${ }^{3}$ Sports Health and Performance Institute, The Ohio State University, Columbus, OH, USA ${ }^{4}$ School of Health and Rehabilitation Sciences, The Ohio State University, Columbus, OH, USA

${ }^{5}$ Division of Sports Medicine, Department of Family Medicine, The Ohio State University, Columbus, OH, USA
}

Keywords: Gait, Quadriceps weakness, Muscle-driven simulation

*Address Correspondence to:

Robert A. Siston

Department of Mechanical and Aerospace Engineering

E305 Scott Laboratory

$201 \mathrm{~W}$ 19th Ave

Columbus, OH 43210-1142 USA

Phone: 614-247-2721

Fax: 614-292-3163

Email: siston.1@osu.edu 


\begin{abstract}
Important activities of daily living, like walking and stair climbing, may be impaired by muscle weakness. In particular, quadriceps weakness is common in populations such as those with knee osteoarthritis (OA) and following ACL injury and may be a result of muscle atrophy or reduced voluntary muscle activation. While weak quadriceps has been strongly correlated with functional limitations in these populations, the important cause-effect relationships between abnormal lower extremity muscle function and patient function remain unknown. The purpose of this study was to explore possible muscle compensation strategies and changes in contribution to support and progression to maintain gait kinematics in response to two sources of quadriceps weakness: atrophy and activation failure. We used muscle-driven simulations to track normal gait kinematics in healthy subjects and applied simulated quadriceps weakness as atrophy and activation failure to evaluate compensation patterns associated with the individual sources of weakness. We found that the gluteus maximus and soleus muscles display the greatest ability to compensate for simulated quadriceps weakness. Relative to the baseline behavior of the muscles, the soleus compensates more to counteract activation deficits in the quadriceps and the gluteus maximus compensates more to counteract atrophy in the quadriceps. The development of this method for estimating the compensation strategies that are necessary to maintain normal gait will enable investigations of the role of muscle weakness in abnormal gait and inform potential rehabilitation strategies to improve such conditions.
\end{abstract}




\section{Introduction}

Muscle strength is important for most activities of daily living, including kneeling, stairclimbing, and walking. In particular, lower extremity muscles perform two main tasks in transporting the body during walking: generation or maintenance of forward velocity and support of the upper body (Winter, 1991). Several studies have investigated how muscles contribute to support and progression during healthy gait (Neptune et al., 2001; Anderson and Pandy, 2003; Neptune et al., 2004; Liu et al., 2006). The gluteus maximus and dorsiflexors have been shown to slow forward progression of the body mass center during early stance while providing vertical support, while the gluteus medius, soleus, and gastrocnemius propel the mass center forward and provide vertical support during late stance (Neptune et al., 2004; Liu et al., 2006). On the other hand, the quadriceps slow forward progression and provide vertical support during early stance (Neptune et al., 2004; Liu et al., 2006; Liu et al., 2008).

Not surprisingly, muscle weakness may impair the ability to perform activities of daily living. Quadriceps weakness is a hallmark impairment following ACL injury (Eastlack et al., 1999; Rudolph et al., 2001) as well as in individuals with knee osteoarthritis (OA) (Hurley and Newham, 1993; Fisher et al., 1997), and is a better determinant of functional limitations and disability in persons with OA than plain radiographic changes (McAlindon et al., 1993; Hurley et al., 1997). Impairment of quadriceps function has been correlated with increased fall risk (Lord et al., 1999), slower walking speed (Gibbs et al., 1996; Connelly and Vandervoort, 1997; Moxley Scarborough et al., 1999), slower speed of sit-to-stand tasks (Moxley Scarborough et al., 1999), and longer time to complete a stair-climbing task (Walsh et al., 1998).

Quadriceps weakness may be a result of muscle atrophy as well as reduced voluntary muscle activation (Hurley and Newham, 1993). Quadriceps strength deficits can be as high as 
$38 \%$ compared to the uninvolved side in individuals with OA (Petterson et al., 2007), 25\% in those with ACL injuries (Williams et al., 2005), and 64\% at 3-4 weeks following a total knee arthroplasty (Mizner et al., 2003). Similarly, quadriceps activation deficits may approach $34 \%$ in certain conditions (Hassan et al., 2001). In noncopers with isolated ACL injury, atrophy and activation failure explained more than $60 \%$ of the variance in quadriceps weakness (Williams et al., 2005). In persons with end-stage OA, both reduced muscle activation and lean muscle crosssectional area contribute to quadriceps muscle weakness, but muscle activation has been found to be the primary determinant of strength in the OA limb (Petterson et al., 2008).

However, the underlying mechanism of how impaired quadriceps function contributes to altered gait remains unknown. Muscle contributions to support and progression have been examined in persons with cerebral palsy and post-stroke hemiparesis (Higginson et al., 2006; Steele et al., 2010), but not in subjects with weak quadriceps, such as with knee OA or following ACL injury. A recent simulation study (van der Krogt et al., 2012) investigated muscle compensations due to weakness by simulating atrophy (decreasing the maximum isometric force parameter) in the muscle model. To our knowledge, no study has investigated the effects of quadriceps activation failure on muscle compensations and changes in contribution to support and progression during gait.

In order to inform rehabilitation interventions that may improve the impairments and function of individuals with weakened quadriceps, it is necessary to establish the cause-effect relationships between abnormal lower extremity muscle function and patient function, as well as the impact of the different sources of weakness (atrophy and activation failure) on gait. The purpose of this study was to develop a method to estimate the muscle compensations and changes in contribution to support and progression which could result in unchanged gait 
kinematics in response to two sources of quadriceps weakness: atrophy and activation failure. We used muscle-driven simulations to track normal gait kinematics in healthy subjects and applied simulated quadriceps weakness as atrophy and activation failure to evaluate muscular compensation patterns associated with the individual sources of weakness. Our long-term goal is to apply this methodology to clinical conditions affecting gait and function to assist in determining targeted physical therapy strategies to improve patient outcomes.

\section{Methods}

\section{Experimental Data}

Seven healthy subjects (4 male and 3 female, Age: $21.9 \pm 2.3$ years, Mass: $72.8 \pm 11.4$ kg, Height: $1.74 \pm 0.08 \mathrm{~m}$ ) provided written informed consent in accordance with the Institutional Review Board of The Ohio State University to participate in this study. Each subject walked at a self-selected speed $(1.31 \pm 0.14 \mathrm{~m} / \mathrm{s})$ while motion data was collected at $150 \mathrm{~Hz}$ using an 8-camera Vicon MX-F40 system and the Point-Cluster Technique (PCT) (Andriacchi et al., 1998). Ground reaction forces were obtained from six force plates (Bertec, Columbus, OH) sampled at $600 \mathrm{~Hz}$. Muscle activation patterns from the gluteus maximus, gluteus medius, rectus femoris, vastus lateralis, biceps femoris, tibialis anterior, medial gastrocnemius, and soleus of both legs were measured with 16-channel surface EMG (Noraxon, Scottsdale, AZ) sampled at $1500 \mathrm{~Hz}$. EMG data were high-pass filtered at $10 \mathrm{~Hz}$, rectified, and RMS smoothed with a 20 ms window.

\section{Modeling and Simulations}

We generated simulations of one gait cycle for each subject using OpenSim software version 2.4 (Delp et al., 2007). A generic musculoskeletal model with 23 degrees of freedom and 92 musculotendon actuators was scaled to match the anthropometry of the individual subjects. 
The dimensions of each body segment in the model were scaled based on relative distances between pairs of markers obtained from motion capture during the static calibration trial and the corresponding virtual marker locations in the model so that the RMS marker error was no more than $2 \mathrm{~cm}$. The experimental gait patterns were reproduced in the scaled model by solving an inverse kinematics problem and a weighted least-squares approach to minimize the differences between the experimental marker locations and the model's virtual marker locations. A residual reduction algorithm (RRA) adjusted model kinematics to resolve dynamic inconsistency between the model kinematics and the ground reaction forces (Delp et al., 2007). Computed muscle control (CMC) (Thelen and Anderson, 2006) was used to calculate the muscle excitations and forces in all lower extremity muscles that produced a coordinated muscle-driven simulation of the subject's gait. The results of RRA and CMC were considered acceptable if the model kinematics differed from experimentally measured kinematics by less than $2^{\circ}$ (or $2 \mathrm{~cm}$ for translations) and if the peak residual forces and moments at the pelvis were less than $20 \mathrm{~N}$ and 50 $\mathrm{Nm}$, respectively. We compared the full-strength simulated muscle activations from CMC to the subject's experimental EMG to ensure that there was agreement between the simulated and experimental muscle activation patterns (Figure 1). An induced acceleration analysis (IAA) was then performed to determine the contributions of individual muscles to the support (vertical acceleration) and progression (horizontal acceleration) of the body mass center (Zajac and Gordon, 1989; Anderson and Pandy, 2003; Hamner et al., 2010). 

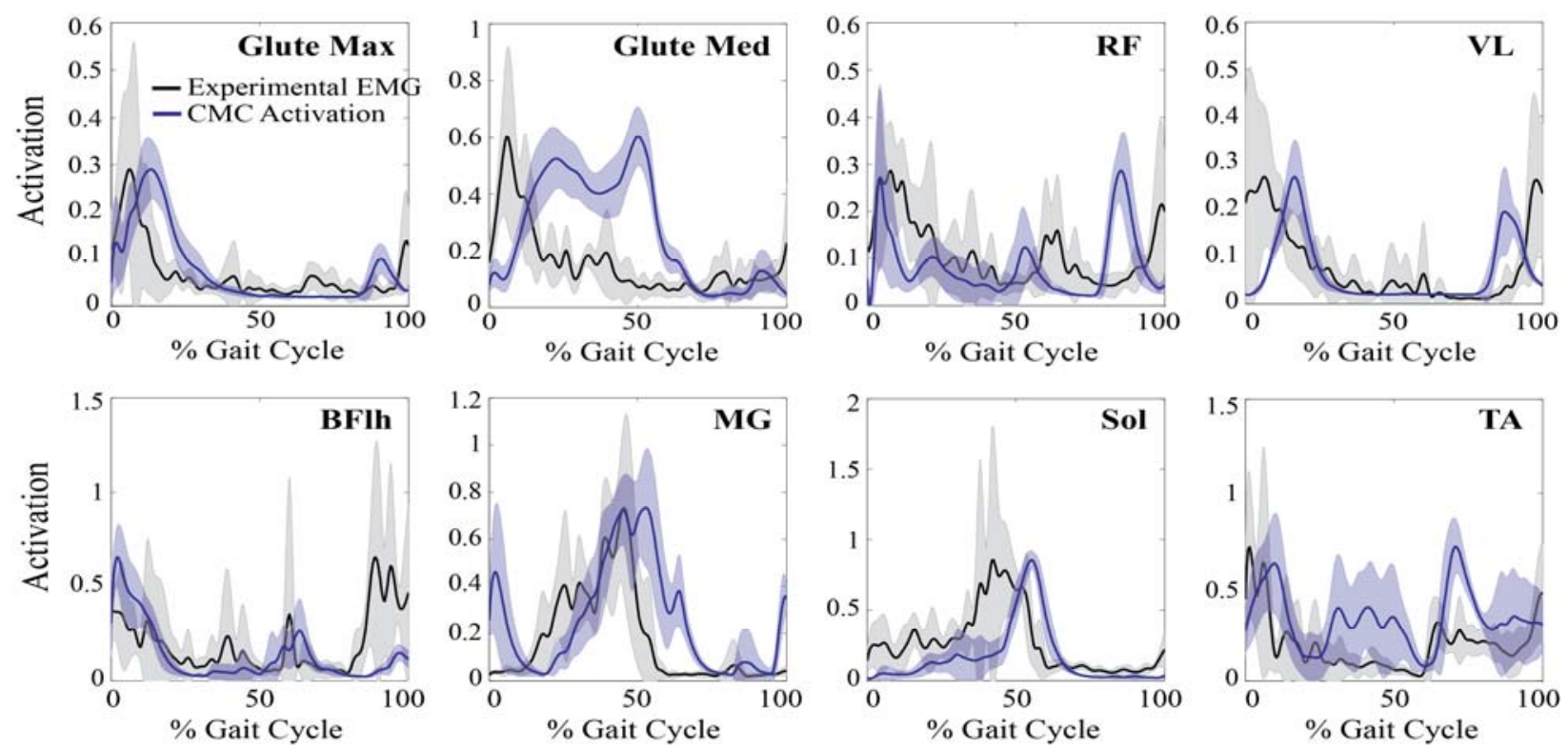

Figure 1: Experimental EMG (black) and simulated muscle activations (blue) averaged over 6 subjects (one subject was excluded due to missing EMG for TA muscle). Shaded areas show one standard deviation. The peak value of the Experimental EMG is normalized to the peak value of the simulated muscle activation.

\section{Simulations with Weakened Quadriceps}

After completing the simulations with the quadriceps at full strength for each subject, we progressively weakened the quadriceps (rectus femoris and vasti) of one stance leg in three ways: 1) decreasing the quadriceps' peak isometric muscle force to $40 \%$ of normal (“Atrophy Only”) to represent a worst-case scenario of quadriceps atrophy, 2) constraining the peak activations of the quadriceps to $65 \%$ of the peak values that were calculated during the full-strength simulation (“Activation Failure Only”) to represent a worst-case scenario of activation deficit and 3) a combination of simulated atrophy and activation failure (“Atrophy + Activation Failure”). We then forced the simulations to track normal gait kinematics, re-calculated muscle forces and contributions to support and progression in the weakened models, and identified the changes in muscle forces and contributions required to maintain normal gait kinematics between the fullstrength simulation and the simulations with the three types of quadriceps weakness. 


\section{Results}

For all subjects and degrees of quadriceps weakness, the simulation was able to successfully track normal gait kinematics. In response to the quadriceps weakness, most muscles in the lower extremity changed their force output and contributions to support and progression (Tables 1-3). Of the major muscle groups investigated, the gluteus maximus and soleus muscles displayed the greatest increases in force and contributions to progression and support in response to simulated quadriceps weakness, indicating the greatest ability to compensate for simulated weakness in the quadriceps muscles during gait. Both muscles increased their force output and contributions to progression and support at different parts of the gait cycle in response to the different types of quadriceps weakness (atrophy and activation failure).

\section{Muscle Forces}

As expected, the quadriceps muscles showed large decreases in force production when they were weakened by simulated atrophy, activation failure, and a combination of these two mechanisms of weakness (Table 1). The medial gastrocnemius showed the next largest decrease in peak force, followed by biceps femoris, while gluteus medius and tibialis anterior showed minimal change in force. The soleus generated more force in mid to late stance compared to the simulation with quadriceps at full strength, with the largest increase in force occurring in response to activation failure of the quadriceps (Table 1 and Figure 2A). The relative compensation was greater in the gluteus maximus than in the soleus (based on \% change from normal), and occurred in early stance and late swing, with the largest increase in force occurring in response to combined atrophy and activation failure of the quadriceps (Table 1 and Figure 2B). 
Table 1: Change in muscle force for each type of simulated quadriceps (RF and Vasti) weakness compared to the full-strength simulation. The largest increases in muscle force occur in the gluteus maximus and soleus muscles. The values are taken from the peak values of the force curves for each muscle during stance averaged over 7 subjects.

\begin{tabular}{|c|c|c|c|c|c|c|}
\hline \multirow[b]{2}{*}{ Muscle } & \multicolumn{2}{|c|}{ Atrophy Only } & \multicolumn{2}{|c|}{ Activation Failure Only } & \multicolumn{2}{|c|}{ Atrophy + Activation Failure } \\
\hline & $\begin{array}{c}\text { Change from } \\
\text { Normal (N) }\end{array}$ & $\%$ change & $\begin{array}{c}\text { Change from } \\
\text { Normal (N) }\end{array}$ & $\%$ change & $\begin{array}{c}\text { Change from } \\
\text { Normal (N) }\end{array}$ & $\%$ change \\
\hline RF (early stance) & -126.7 & -40.4 & -155.8 & -49.7 & -201.7 & -64.3 \\
\hline RF (late stance) & 2.3 & 0.9 & -72.9 & -28.8 & -52.4 & -20.7 \\
\hline Vasti & -68.5 & -9.0 & -27.1 & -3.6 & -128.2 & -16.9 \\
\hline Glute Max & 65.0 & 17.1 & 59.3 & 15.6 & 172.6 & 45.5 \\
\hline Soleus & 130.0 & 7.7 & 239.0 & 14.2 & 196.0 & 11.6 \\
\hline MG & -131.0 & -10.8 & -26.0 & -2.2 & -158.0 & -13.1 \\
\hline BFlh & -21.1 & -6.1 & -3.0 & -0.9 & -18.5 & -5.3 \\
\hline Glute Med & 19.0 & 1.8 & -26.0 & -2.5 & -18.0 & -1.7 \\
\hline TA & -13.6 & -2.7 & 5.6 & 1.1 & -6.7 & -1.3 \\
\hline
\end{tabular}
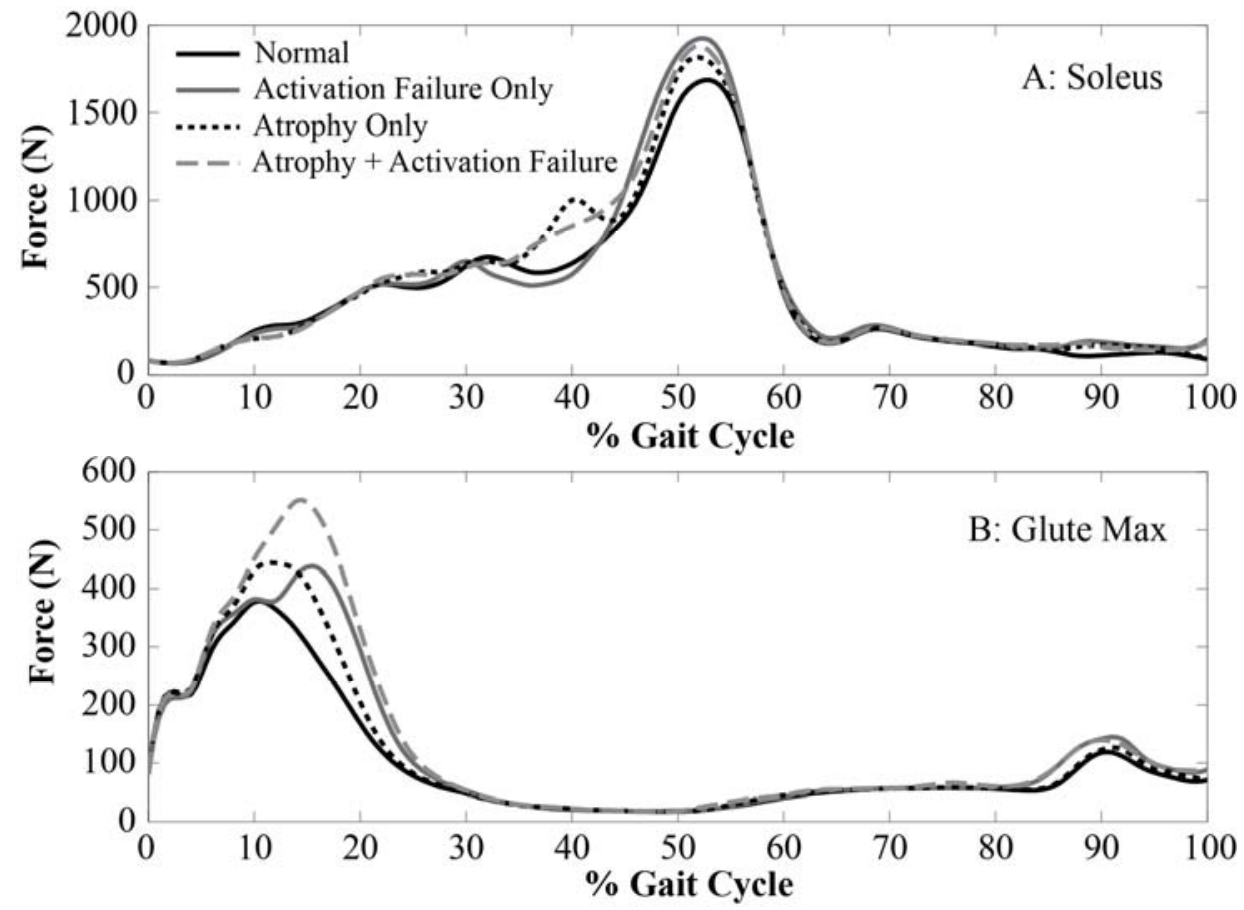

Figure 2: Force generated by the soleus and gluteus maximus muscles in response to the 3 types of simulated quadriceps weakness. (A) The soleus generated more force in mid to late stance in response to activation deficits in the quadriceps, while (B) the gluteus maximus generated more force in early stance to compensate for simulated atrophy + activation failure in the quadriceps. Each line represents the average over 7 subjects. 


\section{Contributions to progression and support}

The quadriceps muscles showed large decreases in their contributions to progression (Table 2) and support (Table 3) when they were weakened by simulated atrophy, activation failure, and a combination of the two types of weakness. Similar to the force results, the medial gastrocnemius showed the largest compensatory decrease in peak contribution to progression and support, followed by biceps femoris, while gluteus medius and tibialis anterior showed minimal change in contributions to progression and support. The soleus showed relatively small changes in contribution to forward progression compared to the simulation with quadriceps at full strength (Table 2 and Figure 3A), and showed greater changes in contribution to vertical support, especially in response to simulated quadriceps activation failure (Table 3 and Figure 3B). The increases in relative contribution to support and progression were generally greater in the gluteus maximus than in the soleus (based on \% change from normal), with the exception of the "Activation Failure Only" case. To maintain a normal gait pattern in response to simulated weakened quadriceps, the gluteus maximus contributed more to slowing forward progression (Table 2 and Figure 4A) and providing vertical support (Table 3 and Figure 4B), especially in response to atrophy of the quadriceps. 
Table 2: Change in muscle contribution to forward progression for each type of simulated quadriceps (RF and Vasti) weakness compared to the full-strength simulation. (A positive change from normal indicates an increase in anterior acceleration of the center of mass, while a negative change from normal indicates an increase in posterior acceleration. A negative \% change indicates a decrease in the absolute magnitude of the acceleration.) The largest increases in contribution to anterior/posterior acceleration occur in the gluteus maximus and soleus muscles. The values are taken from the peak values of the induced acceleration curves for each muscle during stance averaged over 7 subjects.

\begin{tabular}{|c|c|c|c|c|c|c|}
\hline \multirow{2}{*}{ Muscle } & \multicolumn{2}{|c|}{ Atrophy Only } & \multicolumn{2}{c|}{ Activation Failure Only } & \multicolumn{2}{c|}{ Atrophy + Activation Failure } \\
\cline { 2 - 7 } & $\begin{array}{c}\text { Change from } \\
\text { Normal } \\
\left(\mathbf{m} / \mathbf{s}^{2}\right)\end{array}$ & $\%$ change & $\begin{array}{c}\text { Change from } \\
\text { Normal } \\
\left(\mathbf{m} / \mathbf{s}^{2}\right)\end{array}$ & $\begin{array}{c}\text { Change from } \\
\text { Normal } \\
\left(\mathrm{m} / \mathbf{s}^{2}\right)\end{array}$ & \% change \\
\hline RF (early stance) & 0.176 & -38.9 & 0.236 & -52.1 & 0.286 & -63.1 \\
\hline RF (late stance) & 0.012 & -2.5 & 0.139 & -28.4 & 0.118 & -24.1 \\
\hline Vasti & 0.144 & -9.2 & 0.060 & -3.8 & 0.277 & -17.6 \\
\hline Glute Max & $-\mathbf{- 0 . 0 4 6}$ & $\mathbf{1 8 . 9}$ & -0.015 & $\mathbf{6 . 2}$ & $-\mathbf{0 . 0 9 4}$ & $\mathbf{3 8 . 7}$ \\
\hline Soleus & $\mathbf{0 . 0 2 5}$ & $\mathbf{1 . 6}$ & $\mathbf{0 . 1 0 0}$ & $\mathbf{6 . 3}$ & $\mathbf{0 . 0 5 1}$ & $\mathbf{3 . 2}$ \\
\hline MG & -0.126 & -11.3 & -0.005 & -0.4 & -0.128 & -11.4 \\
\hline BFlh & -0.012 & -6.5 & -0.003 & -1.6 & -0.011 & -6.0 \\
\hline Glute Med & -0.005 & 1.0 & 0.006 & -1.2 & 0.0 & 0.0 \\
\hline TA & 0.032 & -1.9 & -0.021 & 1.3 & 0.023 & -1.4 \\
\hline
\end{tabular}


Table 3: Change in muscle contribution to vertical support for each type of simulated quadriceps (RF and Vasti) weakness compared to the full-strength simulation. (A positive change from normal indicates an increase in upward acceleration of the center of mass, while a negative change from normal indicates an increase in downward acceleration. A negative \% change indicates a decrease in the absolute magnitude of the acceleration.) The largest increases in contribution to support occur in the gluteus maximus and soleus muscles. The values are taken from the peak values of the induced acceleration curves for each muscle during stance averaged over 7 subjects.

\begin{tabular}{|c|c|c|c|c|c|c|}
\hline \multirow{2}{*}{ Muscle } & \multicolumn{2}{|c|}{ Atrophy Only } & \multicolumn{2}{c|}{ Activation Failure Only } & \multicolumn{2}{c|}{ Atrophy + Activation Failure } \\
\cline { 2 - 7 } & $\begin{array}{c}\text { Change from } \\
\text { Normal } \\
\left(\mathbf{m} / \mathrm{s}^{2}\right)\end{array}$ & \% change & $\begin{array}{c}\text { Change from } \\
\text { Normal } \\
\left(\mathbf{m} / \mathbf{s}^{2}\right)\end{array}$ & \% change & $\begin{array}{c}\text { Change from } \\
\text { Normal } \\
\left(\mathbf{m} / \mathbf{s}^{2}\right)\end{array}$ & \% change \\
\hline RF (early stance) & 0.281 & -39.3 & 0.359 & -50.2 & 0.450 & -62.9 \\
\hline RF (late stance) & -0.005 & -1.1 & -0.136 & -29.9 & -0.103 & -22.6 \\
\hline Vasti & -0.306 & -8.0 & -0.061 & -1.6 & -0.590 & -15.5 \\
\hline Glute Max & $\mathbf{0 . 2 7 0}$ & $\mathbf{1 5 . 2}$ & $\mathbf{0 . 0 1 9}$ & $\mathbf{1 . 1}$ & $\mathbf{0 . 4 1 8}$ & $\mathbf{2 3 . 5}$ \\
\hline Soleus & $\mathbf{0 . 5 9 8}$ & $\mathbf{8 . 6}$ & $\mathbf{1 . 0 2 2}$ & $\mathbf{1 4 . 8}$ & $\mathbf{0 . 8 2 0}$ & $\mathbf{1 1 . 8}$ \\
\hline MG & -0.639 & -12.2 & -0.070 & -1.3 & -0.822 & -15.7 \\
\hline BFlh & -0.033 & -9.3 & -0.016 & -4.5 & -0.039 & -11.0 \\
\hline Glute Med & -0.024 & -1.2 & 0.001 & 0.0 & -0.058 & -2.8 \\
\hline TA & -0.127 & -2.0 & 0.088 & 1.4 & -0.024 & -0.4 \\
\hline
\end{tabular}



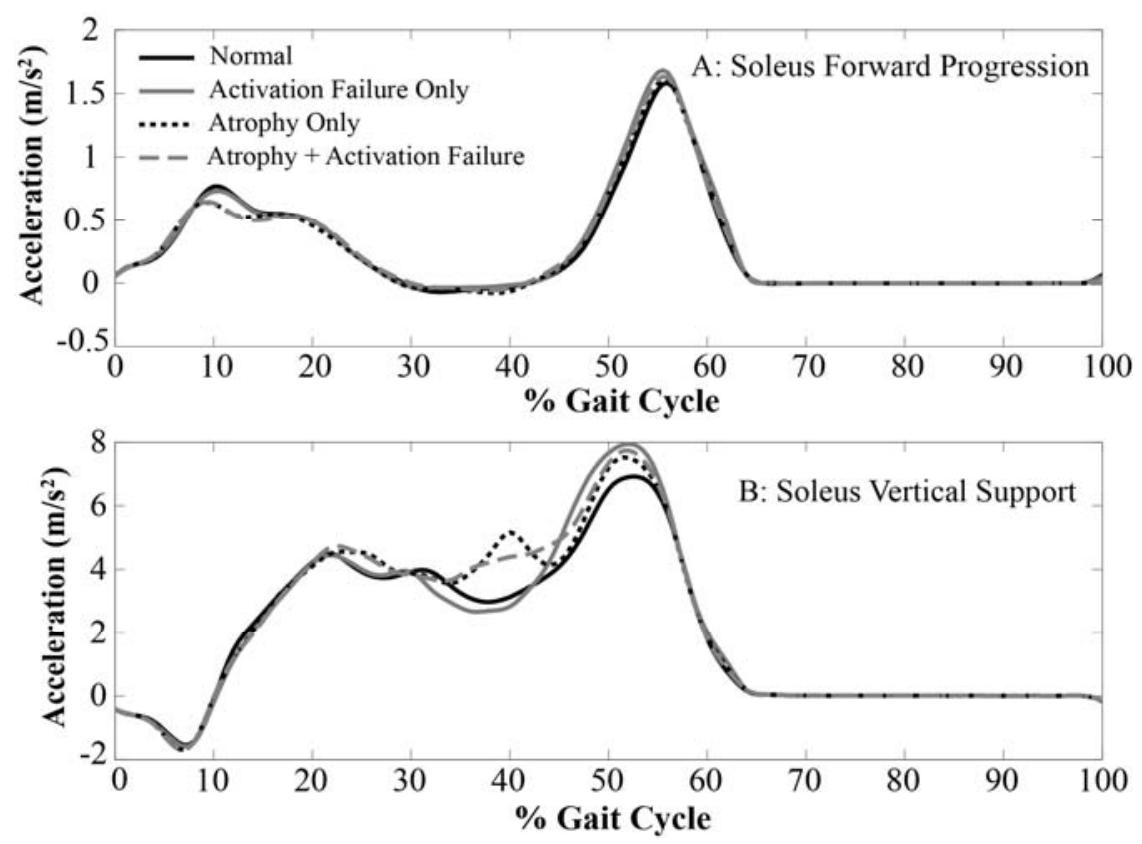

Figure 3: Contribution to (A) forward progression and (B) vertical support by the soleus muscle in response to the 3 types of simulated quadriceps weakness. The soleus contributes more to vertical support than forward progression in response to activation deficits in the quadriceps. Each line represents the average over 7 subjects.
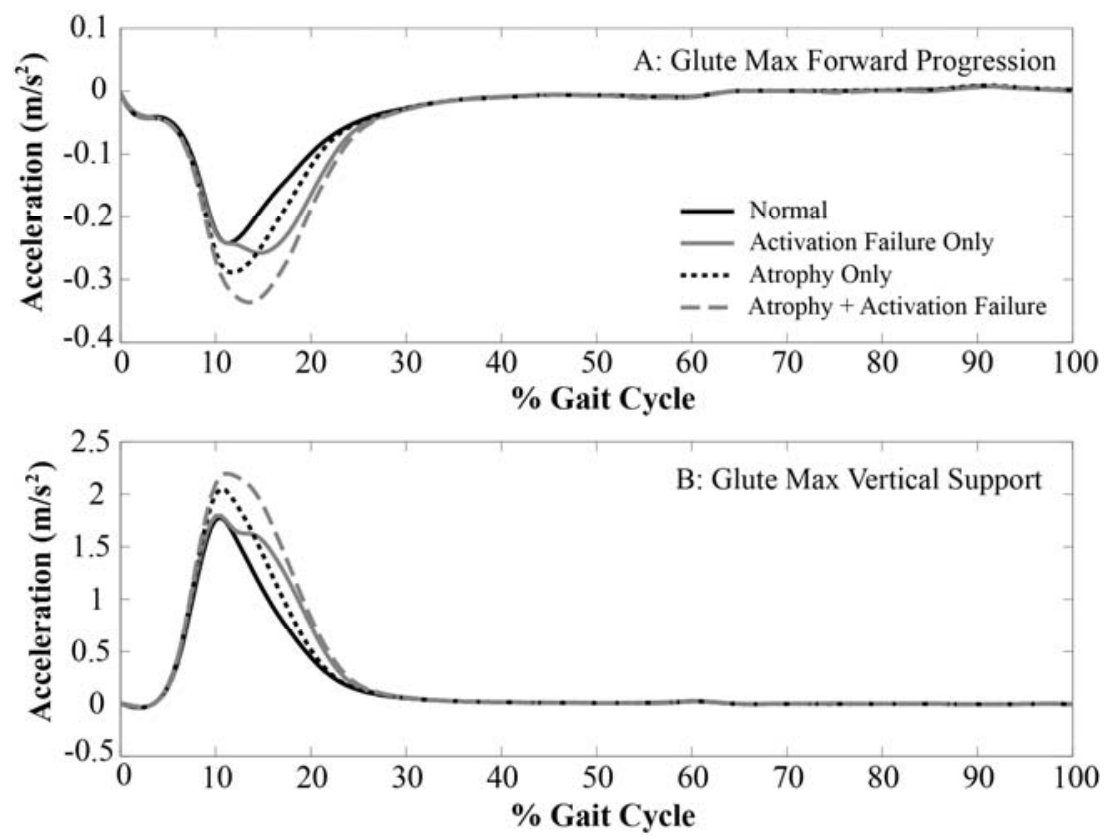

Figure 4: Contribution to (A) forward progression and (B) vertical support by the gluteus maximus muscle in response to the 3 types of simulated quadriceps weakness. Gluteus maximus contributes more to slowing forward progression and maintaining vertical support in response to atrophy and combined atrophy and activation deficits in the quadriceps. Each line represents the average over 7 subjects. 


\section{Discussion}

The purpose of this study was to develop a method to estimate the muscle compensations and changes in contribution to support and progression which could result in unchanged gait kinematics in response to two sources of quadriceps weakness: atrophy and activation failure. To our knowledge, this is the first use of muscle-driven simulations to investigate how lower extremity muscles would compensate for both quadriceps atrophy and activation failure to maintain normal gait kinematics. Our results indicate that the gluteus maximus and soleus muscles show the greatest potential to compensate for weakness in the quadriceps. Furthermore, relative to the baseline behavior of the muscles, the simulation results revealed that greater compensation was shown by the soleus to counteract activation deficits than to counteract atrophy in the quadriceps, indicating that muscle compensation strategies may be different in response to different sources of weakness.

Muscle forces from our simulations compared well with the muscle force results of a previous study of simulated muscle atrophy (van der Krogt et al., 2012). Van der Krogt et al. found similar compensation strategies in other muscles in response to quadriceps weakness, including an increase in force of the gluteus maximus and soleus muscles, and a decrease in force of antagonistic muscles such as the gastrocnemius and biceps femoris. Muscle contributions to support and progression for our full-strength simulations generally agreed with previous studies (Kepple et al., 1997; Neptune et al., 2001; Anderson and Pandy, 2003; Neptune et al., 2004; Liu et al., 2006; Liu et al., 2008). Previous work by Neptune et al. (Neptune et al., 2004) found that the rectus femoris works to accelerate the body forward in late stance. However, in our simulations the rectus femoris contributed to slowing forward progression, which agrees with a more recent simulation study (Liu et al., 2008). 
Interestingly, we found that an increase in muscle force in response to quadriceps weakness did not necessarily translate to an equivalent increase in contribution to support and progression from that muscle. In an effort to relate muscle force and contributions to progression and support, we investigated muscle "potential". The potential of each muscle to contribute to progression and support was calculated by dividing the IAA results for progression and support by the muscle force from CMC (Figure 5). This approach provides a few key insights into our results. First, it helps explain why certain muscles, such as the gluteus maximus and soleus, responded to simulated quadriceps weakness. The quadriceps act to slow progression of the body during the entire stance phase and provide vertical support throughout a large percentage of stance. The only other muscles which provide the potential for consistent "braking" during stance are the gluteus muscles (Figure 5A). The gluteus muscles also contribute to providing vertical support, along with the plantarflexors (gastrocnemius and soleus) (Figure 5B). The soleus is primarily a support muscle, while gastrocnemius assists more with forward progression. Since the loss of quadriceps function affects the body's potential for braking, this may help explain why the soleus, and not the gastrocnemius, responded to simulated quadriceps weakness. In fact, we found that the force and contributions to progression and support decreased in the gastrocnemius. A possible explanation could be that our simulations responded to loss of quadriceps function by decreasing forces in antagonistic muscles such as the gastrocnemius and hamstrings in addition to increasing forces in the gluteus maximus and soleus.

Second, the analysis of muscle potential further highlights the importance of the quadriceps during gait and may provide additional insight into why quadriceps weakness is strongly correlated to sub-optimal functional performance. The quadriceps display the greatest potential of the major muscle groups to consistently slow forward progression during gait. The 
gluteus maximus' potential for slowing forward progression is less than that of the quadriceps. Therefore, there is a limit to how much gluteus maximus could compensate for greater quadriceps weakness than was simulated. For example, at 30\% of the gait cycle, every 1 Newton decrease in force of the vasti would require an increase in force of approximately 4 Newtons from the gluteus maximus to maintain an equivalent contribution to slowing forward progression (Figure 5A).
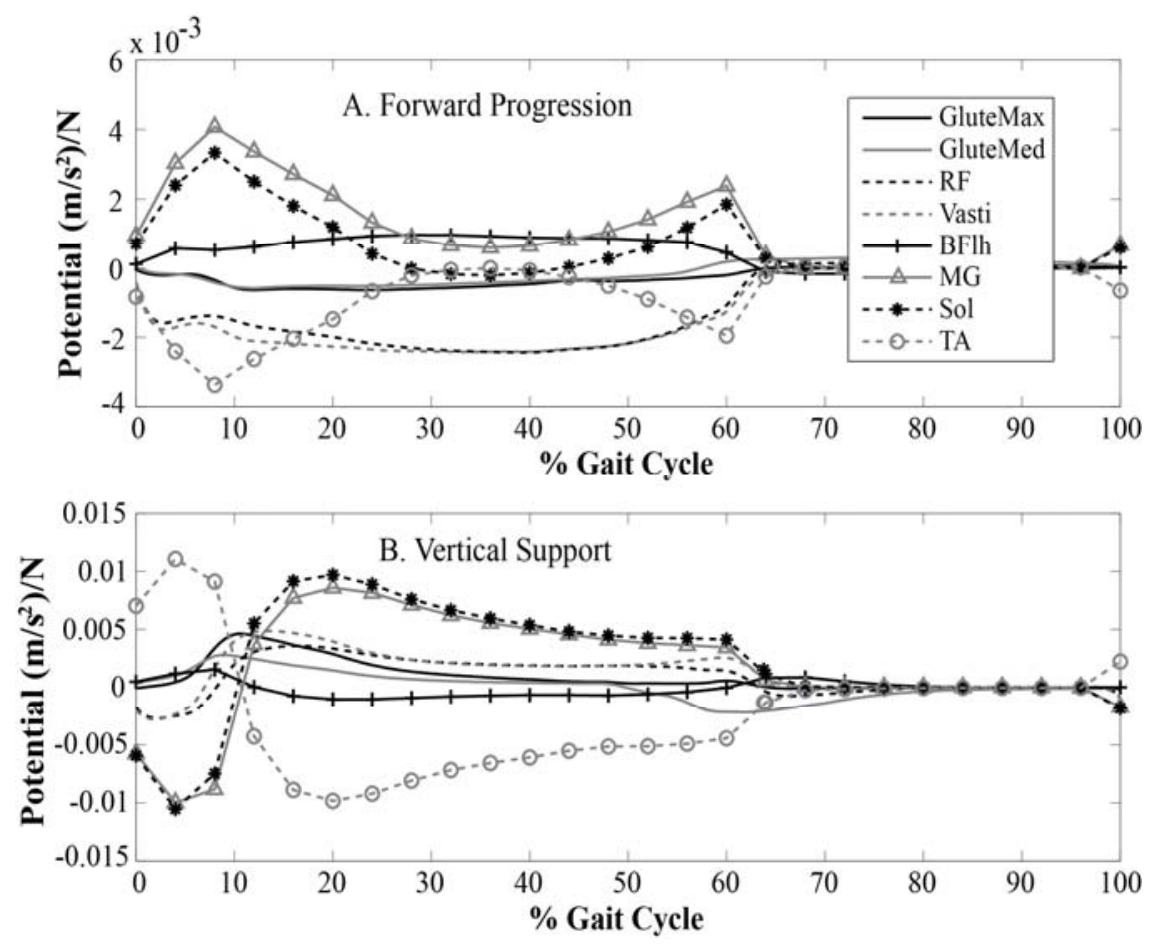

Figure 5: Potential of each of the muscles investigated to contribute to (A) forward progression and (B) vertical support. The potential of the quadriceps (RF and vasti) to contribute to braking is greater than any of the other muscles, with the exception of tibialis anterior (TA) in early stance.

Our results should be considered in light of several limitations. We forced all simulations to track normal gait, but persons with OA or ACL injury often do not use a normal gait pattern (Brinkmann and Perry, 1985; DeVita et al., 1998; Gok et al., 2002; Lewek et al., 2002). The common finding of abnormal gait in persons with pathology suggests that there are 
compensation thresholds beyond which a subject will choose to adapt an altered gait pattern instead of increasing muscle force to maintain normal gait. However, our results offer initial insights to what compensation strategies may be needed for a person with weak quadriceps to maintain normal kinematic patterns during gait. We applied activation failure in the simulations by constraining the peak values of the quadriceps activation to $65 \%$ of the peak values from the full-strength simulation instead of applying an overall constraint on the maximum possible activation of the muscle (assumed to be 1). The peak activation of the quadriceps is typically much less than 1 during gait, so applying the constraint on the maximum activation would not have resulted in any actual activation deficit in the quadriceps during our gait simulations. It is important to note that previous studies of activation failure in the quadriceps have been performed during an experimental maximum isometric contraction. To our knowledge, no study has investigated whether activation failure influences performance of sub-maximal activities such as gait. Therefore, while applying activation deficits to the quadriceps as we did in our simulations may not appropriately reflect in-vivo activation deficits, it represents a key step in investigating the effects of activation failure during walking and utilizes the strengths of simulations in answering “what if?” questions related to muscle function. We also used a generic musculoskeletal model for our simulations, which may not be entirely applicable to populations with weak quadriceps muscles. The quadriceps muscles of persons with OA or following ACL injury have smaller cross-sectional areas and greater activation deficits compared to healthy quadriceps (Hurley et al., 1997; Gur and Cakin, 2003; Lewek et al., 2004; Ikeda et al., 2005; Mizner et al., 2005; Williams et al., 2005; Petterson et al., 2008). Future work should focus on incorporating pathology-specific muscle properties into subject-specific computer models to accurately quantify muscle function during gait in impaired populations. 
Our results provide an important first step in understanding the impact of the sources of quadriceps weakness on dynamic movement gait, as well as the potential compensations by other muscles. While the gluteus maximus and soleus muscles show the greatest potential to compensate for quadriceps weakness, they may not be able to fully compensate for the loss of braking and support provided by the quadriceps. By simulating two different causes of muscle weakness, we were able to elucidate different compensation strategies in response to atrophy and activation deficits. These findings provide novel insights into specific muscle activation patterns that control the various stages of the gait cycle and further investigation is warranted to understand the impact of the sources of weakness in individuals with pathology to determine their compensation strategies that can then be evaluated through clinical interventions.

\section{Acknowledgements}

The authors would like to acknowledge Mike McNally for his assistance with data collection and Jay Young, Molly Mollica, Michelle Cullen, and Laura Henkel for their help with data

processing. Funding for this research was provided by the NSF Graduate Research Fellowship program and the Ohio State University Graduate Fellowship program to Julie Thompson.

\section{Conflict of interest statement}

None of the authors have a conflict of interest regarding the contents of this manuscript.

\section{References}

Anderson, F.C. and Pandy, M.G., 2003. Individual muscle contributions to support in normal walking. Gait Posture 17, 159-69.

Andriacchi, T.P., Alexander, E.J., Toney, M.K., Dyrby, C. and Sum, J., 1998. A point cluster method for in vivo motion analysis: applied to a study of knee kinematics. J Biomech Eng 120, 743-9.

Brinkmann, J.R. and Perry, J., 1985. Rate and range of knee motion during ambulation in healthy and arthritic subjects. Phys Ther 65, 1055-60. 
Connelly, D.M. and Vandervoort, A.A., 1997. Effects of detraining on knee extensor strength and functional mobility in a group of elderly women. J Orthop Sports Phys Ther 26, 340-6.

Delp, S.L., Anderson, F.C., Arnold, A.S., Loan, P., Habib, A., John, C.T., Guendelman, E. and Thelen, D.G., 2007. OpenSim: open-source software to create and analyze dynamic simulations of movement. IEEE Trans Biomed Eng 54, 1940-50.

DeVita, P., Hortobagyi, T. and Barrier, J., 1998. Gait biomechanics are not normal after anterior cruciate ligament reconstruction and accelerated rehabilitation. Med Sci Sports Exerc 30, 1481-8.

Eastlack, M.E., Axe, M.J. and Snyder-Mackler, L., 1999. Laxity, instability, and functional outcome after ACL injury: copers versus noncopers. Med Sci Sports Exerc 31, 210-5.

Fisher, N.M., White, S.C., Yack, H.J., Smolinski, R.J. and Pendergast, D.R., 1997. Muscle function and gait in patients with knee osteoarthritis before and after muscle rehabilitation. Disabil Rehabil 19, 47-55.

Gibbs, J., Hughes, S., Dunlop, D., Singer, R. and Chang, R.W., 1996. Predictors of change in walking velocity in older adults. J Am Geriatr Soc 44, 126-32.

Gok, H., Ergin, S. and Yavuzer, G., 2002. Kinetic and kinematic characteristics of gait in patients with medial knee arthrosis. Acta Orthop Scand 73, 647-52.

Gur, H. and Cakin, N., 2003. Muscle mass, isokinetic torque, and functional capacity in women with osteoarthritis of the knee. Arch Phys Med Rehabil 84, 1534-41.

Hamner, S.R., Seth, A. and Delp, S.L., 2010. Muscle contributions to propulsion and support during running. J Biomech 43, 2709-16.

Hassan, B.S., Mockett, S. and Doherty, M., 2001. Static postural sway, proprioception, and maximal voluntary quadriceps contraction in patients with knee osteoarthritis and normal control subjects. Ann Rheum Dis 60, 612-8.

Higginson, J.S., Zajac, F.E., Neptune, R.R., Kautz, S.A. and Delp, S.L., 2006. Muscle contributions to support during gait in an individual with post-stroke hemiparesis. J Biomech 39, 1769-77.

Hurley, M.V. and Newham, D.J., 1993. The influence of arthrogenous muscle inhibition on quadriceps rehabilitation of patients with early, unilateral osteoarthritic knees. Br J Rheumatol 32, 127-31. 
Hurley, M.V., Scott, D.L., Rees, J. and Newham, D.J., 1997. Sensorimotor changes and functional performance in patients with knee osteoarthritis. Ann Rheum Dis 56, 641-8.

Ikeda, S., Tsumura, H. and Torisu, T., 2005. Age-related quadriceps-dominant muscle atrophy and incident radiographic knee osteoarthritis. J Orthop Sci 10, 121-6.

Kepple, T.M., Siegel, K.L. and Stanhope, S.J., 1997. Relative contributions of the lower extremity joint moments to forward progression and support during gait. Gait \& Posture 6, 1-8.

Lewek, M., Rudolph, K., Axe, M. and Snyder-Mackler, L., 2002. The effect of insufficient quadriceps strength on gait after anterior cruciate ligament reconstruction. Clin Biomech (Bristol, Avon) 17, 56-63.

Lewek, M.D., Rudolph, K.S. and Snyder-Mackler, L., 2004. Quadriceps femoris muscle weakness and activation failure in patients with symptomatic knee osteoarthritis. J Orthop Res 22, 110-5.

Liu, M.Q., Anderson, F.C., Pandy, M.G. and Delp, S.L., 2006. Muscles that support the body also modulate forward progression during walking. J Biomech 39, 2623-30.

Liu, M.Q., Anderson, F.C., Schwartz, M.H. and Delp, S.L., 2008. Muscle contributions to support and progression over a range of walking speeds. J Biomech 41, 3243-52.

Lord, S.R., Rogers, M.W., Howland, A. and Fitzpatrick, R., 1999. Lateral stability, sensorimotor function and falls in older people. J Am Geriatr Soc 47, 1077-81.

McAlindon, T.E., Cooper, C., Kirwan, J.R. and Dieppe, P.A., 1993. Determinants of disability in osteoarthritis of the knee. Ann Rheum Dis 52, 258-62.

Mizner, R.L., Petterson, S.C., Stevens, J.E., Vandenborne, K. and Snyder-Mackler, L., 2005. Early quadriceps strength loss after total knee arthroplasty. The contributions of muscle atrophy and failure of voluntary muscle activation. J Bone Joint Surg Am 87, 1047-53.

Mizner, R.L., Stevens, J.E. and Snyder-Mackler, L., 2003. Voluntary activation and decreased force production of the quadriceps femoris muscle after total knee arthroplasty. Phys Ther 83, 359-65.

Moxley Scarborough, D., Krebs, D.E. and Harris, B.A., 1999. Quadriceps muscle strength and dynamic stability in elderly persons. Gait Posture 10, 10-20.

Neptune, R.R., Kautz, S.A. and Zajac, F.E., 2001. Contributions of the individual ankle plantar flexors to support, forward progression and swing initiation during walking. J Biomech 34, 1387-98. 
Neptune, R.R., Zajac, F.E. and Kautz, S.A., 2004. Muscle force redistributes segmental power for body progression during walking. Gait Posture 19, 194-205.

Petterson, S.C., Barrance, P., Buchanan, T., Binder-Macleod, S. and Snyder-Mackler, L., 2008. Mechanisms underlying quadriceps weakness in knee osteoarthritis. Med Sci Sports Exerc 40, 422-7.

Petterson, S.C., Raisis, L., Bodenstab, A. and Snyder-Mackler, L., 2007. Disease-specific gender differences among total knee arthroplasty candidates. J Bone Joint Surg Am 89, 2327-33.

Rudolph, K.S., Axe, M.J., Buchanan, T.S., Scholz, J.P. and Snyder-Mackler, L., 2001. Dynamic stability in the anterior cruciate ligament deficient knee. Knee Surg Sports Traumatol Arthrosc 9, 62-71.

Steele, K.M., Seth, A., Hicks, J.L., Schwartz, M.S. and Delp, S.L., 2010. Muscle contributions to support and progression during single-limb stance in crouch gait. J Biomech 43, 2099-105.

Thelen, D.G. and Anderson, F.C., 2006. Using computed muscle control to generate forward dynamic simulations of human walking from experimental data. J Biomech 39, 1107-15.

van der Krogt, M.M., Delp, S.L. and Schwartz, M.H., 2012. How robust is human gait to muscle weakness? Gait Posture 36, 113-9.

Walsh, M., Woodhouse, L.J., Thomas, S.G. and Finch, E., 1998. Physical impairments and functional limitations: a comparison of individuals 1 year after total knee arthroplasty with control subjects. Phys Ther 78, 248-58.

Williams, G.N., Buchanan, T.S., Barrance, P.J., Axe, M.J. and Snyder-Mackler, L., 2005. Quadriceps weakness, atrophy, and activation failure in predicted noncopers after anterior cruciate ligament injury. Am J Sports Med 33, 402-7.

Winter, D.A., 1991. The Biomechanics and Motor Control of Human Gait: Normal, Elderly and Pathological. University of Waterloo Press, Waterloo.

Zajac, F.E. and Gordon, M.E., 1989. Determining muscle's force and action in multi-articular movement. Exerc Sport Sci Rev 17, 187-230. 